\title{
A Semi-Independent Structure for the Architectural Design of the Converged Scenario for Integrated Hybrid PON with Wireless Technologies for Next Generation Broadband Access Networks
}

\author{
N. Moradpoor \\ School of Science, Engineering and Technology \\ University of Abertay Dundee \\ Dundee, UK \\ e-mail: n.moradpoor@abertay.ac.uk
}

\begin{abstract}
Optical and wireless technology integration schemes merge the high-speed and high-capacity of the optical networks with the low-cost, wide-coverage and mobility features of wireless counterparts for Subscriber Stations (SSs). It is also financially viable for the telecommunication service providers, particularly in rural areas. In order to successfully integrate the two technologies, there are some technical concerns in terms of Architectural aspects, Physical Layer features and Media Access Control (MAC) related issues. This paper is mainly focused on the analysis of the key topics in architectural design of the converged scenario such as ETE module specifications as well as the MAC-related issues such as ETE resource allocations between the hybrid Passive Optical Networks (hybrid PONs) and wireless technology. A semi-independent structure for the architectural design of the converged protocol is proposed in this paper along with the ETE functional modules from the Optical Line Terminal (OLT) at the far-end Central Office (CO) all the way to the SSs at the front-end customer premises. The ETE key functional modules, which provide the MAC-related issues for the converged protocol such as ETE resource requests and allocations, are also highlighted.
\end{abstract}

Keywords: optical and wireless technology integration, converged architecture, Media Access Control (MAC), resource allocations

\section{INTRODUCTION}

PON [1], [2], and [3] is the most promising candidate among optical access solutions in terms of maintainability and robustness. There have been various efforts on PON multiplexing techniques such as Time Division Multiplexing (TDM), Wavelength Division Multiplexing (WDM) and Code Division Multiplexing (CDM). TDMPON reduces the cost per subscriber and has inexpensive network components as it requires only one transmitter in the Optical Line Terminal (OLT), as well as only one type of transmitter in Optical Network Units (ONUs) [4]. However, TDM-PON sacrifices the maximum available bandwidth per subscriber and limits the number of supported subscribers up to 32 [5]. On the other hand, WDM-PON provides multiple wavelength channels with a good security and protocol transparency which offers higher bandwidth and supports more subscribers [6]. In WDMPON [17], a WDM transmitter is the most critical component where the associated transmitter should be precisely aligned with the allocated channel [5]. Unlike
TDM-PON, in WDM-PON, the OLT needs to have an array of transmitters with one transmitter for each ONU. Every ONU also needs to have a wavelength-specific laser. Generally speaking, although PON has been viewed as an attractive solution for the "first/last mile" bandwidth bottleneck problem, extending fibre-based infrastructures of PON to the rural area is either too costly or inaccessible. Moreover, PON is unable to provide wireless access services.

World Wide Interoperability for Microwave Access (WiMAX, IEEE 802.16 [7]) standard comes into play as a wireless matching part for PON technology. WiMAX aims to reduce the equipment, operation and maintenance costs and capable of providing the low-cost, wide coverage, fixed and mobile broadband access connections with the QoS provisioning scheme [8]. It also provides wireless access services for rural areas where the development of copperbased technologies or fibre-based broadband is too expensive or inaccessible. However, WiMAX copper-based backhaul technology is still a controversial issue. This is the point where optical and wireless technology integrations come into play. PON can be used as a scalable and costaware solution for WiMAX backhaul problem whereas WiMAX can extend PON infrastructure to rural areas with relatively lower cost.

In order to successfully integrate the optical and wireless technologies, there are some challenging issues that need to be addressed efficiently and effectively in order to provide the smooth End-To-End (ETE) technology integrations. To the best of our knowledge, the traditional single channel TDM-PON has been addressed in most of the existing work related to the optical and wireless integration scenarios. Therefore, we are motivated to combine the wavelength routing and high-capacity of WDM-PON, the power-splitting and lower-cost of TDMPON with high coverage and mobility features of the wireless counterpart for the integrated scenario. To achieve this goal, an architectural design of the converged protocol is proposed in this paper along with the required ETE functional modules and ETE key functional modules.

The remainder of this paper is prepared as follows. In Section II, the existing work related to the optical and wireless technology integrations is briefly discussed. Section III discusses the proposed architectural design of 
the converged protocol flowed by Sections IV, which includes the conclusions and work in progress.

\section{RELATED WORK}

As the work in this paper is mostly related to the Architectural aspects of the converged networks, the previous works related to this aspect are discussed as follows.

In [9], the authors proposed four architectures for integration of EPON, and WiMAX termed Independent Architecture, Hybrid Architecture, Unified ConnectionOriented Architecture and Microwave-over-Fiber Architecture. Independent architecture is the simplest approach to implement where each part of the converged network works independently from one another. In Hybrid Architecture, the ONU and BS are integrated in a single box termed ONU-BS in which each part of the converged network has full information about the other part. In Unified Connection-Oriented Architecture, the authors proposed a new integration scheme in which the MAC layer of EPON has been modified in order to support the connectionoriented services. In Microwave-over-Fiber Architecture, each remote node includes a single ONU and a dump antenna, responsible for relaying a WiMAX radio signal from and to its associated microcell. In [10], the authors proposed Hybrid Wireless Optical Broadband Access Network (WOBAN) in order to save the network design and deployment costs. The optimal placement of the ONU was investigated in WOBAN by taking into account several optimization algorithms. In [11], the authors proposed the hybrid optical wireless access network that consists of reconfigurable optical backhaul and wireless mesh networks (WMNs). They have also proposed an integrated routing algorithm that can dynamically choose the optimum route in order to balance the total network load when the congestion happens to the WMN part. In [12], the authors proposed Optical-Optical-Wireless (OOW) architecture for the EPON-WiMAX integration scenario in which OLT is connected to a group of sub-OLTs instead of the ONUs. In [13], the authors presented the concepts of the Virtual ONU-BS (VOB) which includes EPON-ONU, WiMAX-BS and a WiMAX-EPON Bridge (WE-Bridge). In [14], the authors demonstrated the major building components of the ONU-BS layer two integrations, including the Protocol Processing, Packet Classification, DBA scheduling and QoS modules. For more related work, please refer to [15] and [16].

In this paper, a semi-independent structure for the architectural design of the converged protocol is proposed. It is aimed to employ the optical and wireless components and infrastructures, which are available in the market, and then considering adding the extra functional modules with no need for full replacements. This is the main difference between the work in this paper and the Independent structure work which was proposed in [9]. Next section provides a detailed description about the proposed architectural design.

\section{Proposed ARChitectural Design of the CONVERGED PROTOCOL}

In this section, the proposed semi-independent structure for the architectural design of the converged scenario is discussed in detail.

\section{A. Propsoed ETE Functional Modules}

The proposed ETE functional modules include all the modules, which need to be provided inside OLT, ONUs, BSs, APs as well as the WLAN subscriber stations in order to develop an infrastructure for the multi-channel PON convergence with wireless technologies of Next-Generation Networks (NGNs), Figure.1. As Figure.1 reveals, all the functional modules are marked with arrows, which run periodically and in sequence one after another. Based on the dynamic/static nature of the converged scenario, the functional modules can run only either once or once per service cycle. A service cycle is the maximum time duration that a given ONU or BS can wait before it gets a chance to transmit the buffered data in the directions of the OLT or the ONU, both respectively. All the proposed functional modules, Figure.1, are detailed in order as follows.

1. OLT-ONU and ONU-OLT $->$ Auto-Discovery modules: these modules are employed in order to discover and then register the existing ONUs into the OLT's optical domain. The fundamental waveband architecture, MAC address as well as the RTT delay will be identified for each ONU at this stage by employing the MPCP Extension protocol. MPCP was developed by IEEE 802.3ah [3] in order to settle the real-time bandwidth negotiations in traditional TDM-based PON. While the MPCP provides time-slot assignment features, the MPCP Extension [2] adds wavelength assignment supports into the conventional MPCP protocol which is enabling the OLT to schedule transmission to and from a given ONU on any available and supported wavelengths. In this stage, the first three MPCP Ext. control messages termed REGISTER REQUEST, REGISTER and REGISTER ACKNOWLEDGE will be executed in order to register the available ONUs into the OLT's optical domain.

2. OLT-> wavelength / bandwidth allocation modules: OLT-> wavelength allocation module, module 2.a, allocates a wavelength (channel) to a given ONU based on the ONU's waveband architecture, which were collected by module 1, OLT's supported and available wavelengths as well as the network load per channel. Wavelength allocation module needs a wavelength allocation algorithm to be implemented and installed in module 2.a placed inside the OLT. Moreover, the OLT allocates the bandwidth to a given ONU employing the implemented bandwidth allocation algorithm (static/dynamic) seated inside module 2.c. In dynamic bandwidth allocation, the bandwidth will be granted based on the ONU's actual need, which is reported in every service cycle and processed by module 2.b, the OLT's available bandwidth and also the DBA boundaries. OLT-> GATE generator modules (wavelength/bandwidth) will be executed immediately after module 2.c in order to occupy the decided channel ID, which is the output from module 2.a, as well as the allocated bandwidth, transmission start-time and transmission duration, which are the outputs from module 2.c, inside the MPCP Ext. GATE protocol. The generated GATE message will broadcast immediately to all ONUs. Generally speaking, the MPCP Ext. REPORT and MPCP Ext. GATE messages are employed through module 2 in this stage.

3. The ONU-BS and BS-ONU -> Auto-Discovery modules: these modules are employed in order to distinguish the number of BSs in the ONU's optical domain. Information such as: Round Trip Time (RTT), individual MAC address as well as the waveband 
boundaries will be collected from each BS during this stage. The MPCP Ext. protocol, particularly REGISTER REQUEST, REGISTER and REGISTER ACKNOWLEDGE messages are employed for such a purpose. This information will be employed for future wavelength/bandwidth allocations from a given ONU to the correspondent BSs. The functionalities of the ONUBS and BS-ONU -> Auto-Discovery modules are the same as the module 1 .

4. WLAN users-> traffic generator module: This module, which is the only source to generate the network traffic, produces traffic with different priorities as soon as WLAN users start their Internet connections.

5. AP-> Classifier module: User traffic, which is generated by module 4 , is immediately received by the associated AP and passed through the AP Classifier module. It classifies different traffic types by buffering them based on the traffic priorities, e.g. voice traffic, video traffic and email traffic as first, second and third priorities all correspondingly.

6. AP-> REQUEST generator module: as soon as the AP's buffers are occupied by the data traffic from the associated WLAN users, the AP REQUEST generator module sends bandwidth requests to the associated BS. Bandwidth requests from a given AP will be issued per priority queue and will be classified based on the priority queue and the requested bandwidth inside a given BS.

7. BS-> PULLING module: It pulls the AP bandwidth requests and goes to the next module immediately.

8. BS- $>$ REPORT generator module: The BS needs to send the regular MPCP Ext. REPORT messages in order to ask for bandwidth from the associated ONU. The BS generalises the different bandwidth requests received from the associated APs based on the connection ID, requested amount of bandwidth (bits) as well as the Class of Service $(\mathrm{CoS})$ inside Bandwidth Request Table (BRT). The REPORT generator module is responsible for tailoring the requested bandwidth (bits) from all APs associated with a given BS at the end of the latest received MPCP Ext. GATE message from the ONU. The BS then needs to wait for the next MPCP Ext. GATE message to arrive before sending any data to the ONU. The MPCP Ext. can report up to eight different priority queues inside each single MPCP Ext. REPORT message.

9. ONU-> REPORT generator and GATE processor (wavelength/bandwidth) modules: The ONU-> REPORT generator module, module 9.a, is provided inside the ONU for the purpose of bandwidth requests from the OLT which is capable of reporting up to eight priority queues in every service cycle. The ONU-> GATE processor (wavelength/bandwidth) modules, modules 9.b and 9.c, are also presented in order to find out the wavelength/bandwidth allocation decisions from the OLT, including channel ID, the amount of allocated bandwidth (channel portion), transmission start-time and transmission duration in every service cycle.

10. ONU-> wavelength/bandwidth allocation modules and ONU-> REPORT processor module: These features require the wavelength/bandwidth allocation algorithms to be installed inside the ONU, inside modules 10.a and 10.c respectively, in order to allocate the wavelength and bandwidth, respectively, from a given ONU to the associated BSs. ONU-> REPORT processor module, module 10b, also helps the wavelength/bandwidth allocation processes by processing the bandwidth requests received from the associated BSs in every service cycle.

11. ONU-> GATE generator modules (wavelength /bandwidth): The wavelength/bandwidth allocation decisions, which are decided by modules 10a and 10.c, respectively, reside inside the MPCP Ext. GATE messages by the ONU-> GATE generator modules (wavelength /bandwidth) and broadcast immediately to all BSs. The MPCP Ext. GATE messages carry the allocated channel ID, allocated bandwidth, transmission start-time, and transmission duration for all the associated BSs of a given ONU in a given service cycle.

12. BS-> GATE processor modules (wavelength /bandwidth): The wavelength and bandwidth allocation decisions, which are generated by module 11 and received at a given $\mathrm{BS}$ in the format of the MPCP GATE message, need to be processed by the GATE processor module installed inside a given BS, modules 12.a and 12.b, respectively. First, the MAC address should be checked; obviously, non-match MPCP Ext. GATE messages will be discarded as they are meant to be for other BSs in the same optical domain. If the MAC address is matched, the wavelength ID, transmission start-time and transmission duration will be captured in order to schedule the transmission of the data traffic from a given BS over the allocated channel.

13. BS-> GRANT generator module: This module distributes the latest receive GATE message among all the bandwidth requests, which have previously arrived from the associated APs on a given BS. The bandwidth requests were pulled from the APs previously and stored inside the BS BRT during module 7 and 8 executions. The GRANT messages will be received by the APs after the execution of the BS-> GRANT generator module, and they will be sent to the SCHEDULER inside a given AP (module 14) immediately.

14. AP-> SCHEDULER module: This is the only module inside a given AP which has access to different priority queues and decides which queue, for how long and in what order should start sending traffic.

15. BS-> QoS mapping module: This module maps traffic types with different QoS requirements from the wireless domain into the optical domain in order to provide smooth ETE data transmission. For instance, WiMAX supports five service classes termed UGS, rtPS, ertPS, nrtPS and BE while an EPON supports three service classes termed EF, AF and BE. Therefore, some mapping techniques need to be provided in order to map different classes of services travelling between the two technologies.

16. BS- $>$ Scheduler module: This module specifies, which queue in what order and for how long should start sending data traffic to the ONU. BS-> Scheduler module is executed immediately after module 15 .

17. ONU-> ROUTING module: The data traffic which arrives from a given BS on a given ONU will be forwarded to the OLT immediately using module 17 . This is the traffic which was originally generated from module 4 .

18. OLT-> ROUTING module: Finally, data bursts, which arrived from WLAN users respectively on a given $\mathrm{AP}, \mathrm{BS}$, ONU and OLT, will be directly forwarded to the Internet using the OLT-> ROUTING module.

Table I. summarises the ETE delays that a given packet experiences during the journey from a WLAN subscriber station

to the

OLT. 
OLT (CO)

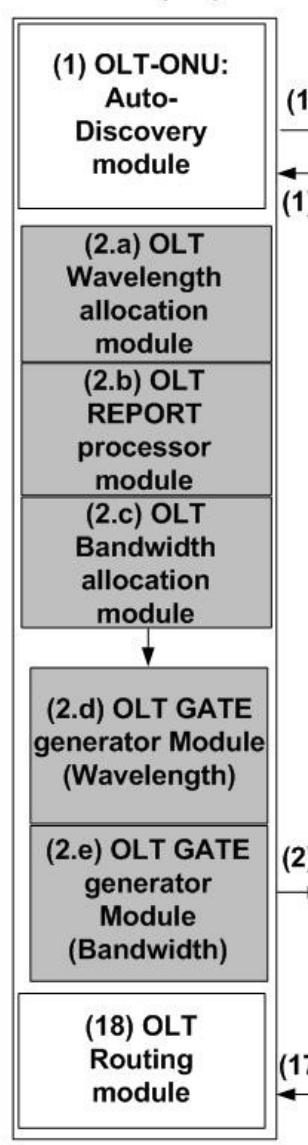

Advanced ONU

(1) ONU-
OLT: Auto-
Discovery
module

module
(1)

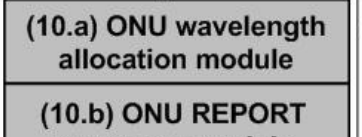

processor module

(10.c) ONU Bandwidth

allocation module

$$
\checkmark
$$

(11.a) ONU GATE

generator Module

(Wavelength)

(11.b) ONU GATE

generator Module

(Bandwidth)

(9.a) ONU REPROT generator module

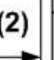

(9.b) ONU GATE

processor module

(wavelength)

(9.c) ONU GATE

processor module

(bandwidth)

(17) ONU

Routing module
Advanced BS

(3) BS-

ONU: Auto-

Discovery module

(3)

(8)

(8) BS REPORT generator module

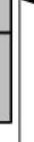

(11)

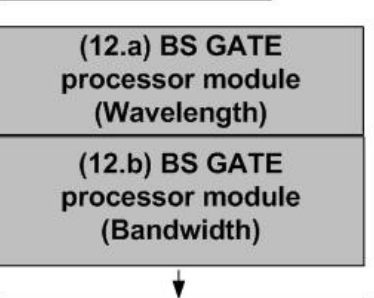

(13) BS GRANT generator module

Figure.1 Proposed functional modules for the converged scenario
AP

WLAN users

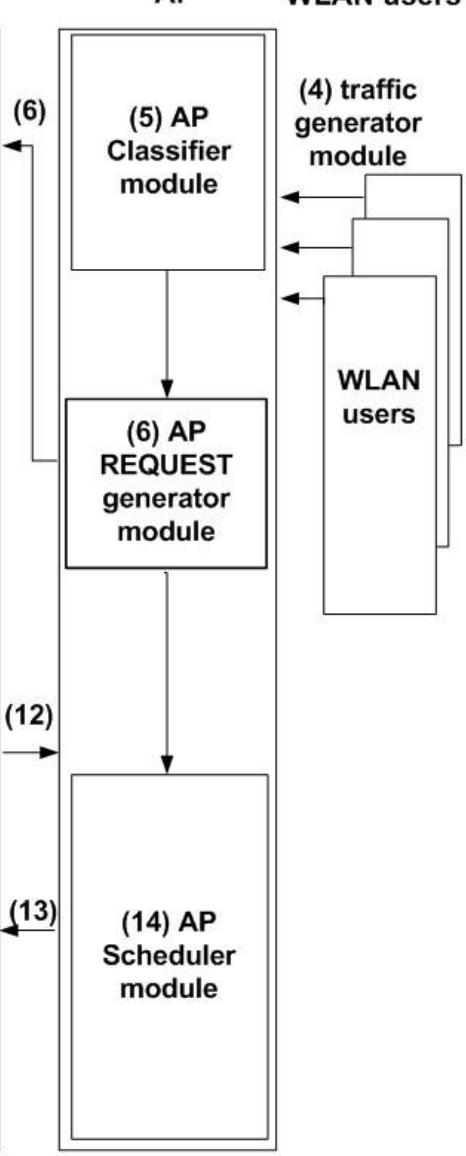

The Next section discusses the proposed key functional modules, among module 1 to module 18 (Figure.1) as follows.

\section{B. Propsoed ETE Key Functional Modules}

In Figure.1, the key functional modules (module $2,8,9,10,11$ and 12) are specified from the other modules by colour. They are termed key functional modules as they have key roles as part of the wavelength/bandwidth allocations from the OLT to the ONUs and then from a given ONU to the associated BSs. The key functional modules can run periodically e.g. once per service cycle or run only once at the beginning of the network setup stage. This is how the dynamic or static nature of the ETE resource allocations will be formed. For instance, if the OLT allocates a channel to a given ONU during the AutoDiscovery stages only once, module 2.a runs only once. However, if the allocated channel from the OLT to a given ONU is changed during the simulation run time based on e.g. channel load or channel availability, module 2.a will need to run periodically, e.g. once per service cycle, in order to provide the dynamic wavelength allocations for the associated ONUs. The proposed key functional modules are detailed in Table II.

\section{CONCLUSION AND WORK IN PROGRESS}

In this paper, an architectural design of the converged protocol was proposed, which embraced the way optical and wireless technologies could be connected to each other. The proposed ETE functional modules from the OLT all the way to the WLAN SSs were fully detailed. It is then followed by the proposed key functional modules which were provided in order to progress the ETE wavelength/bandwidth allocation over the converged scenario. These modules were called key functional modules as they have key roles as part of the ETE resource allocations over the integrated scenario.

The work in progress for this paper is to evaluate the key functional modules from the proposed architectural design of the converged scenario in terms of wavelength/bandwidth allocation algorithms, different traffic patterns and load values under conducted simulation scenarios.

TABLE I. ETE DELAYS FOR THE INTEGRATED SCENARIO

\begin{tabular}{|l|l|}
\hline WLAN & Media access delay \\
\hline AP & $\begin{array}{l}\text { Classification delay, Queuing delay, Media access delay, } \\
\text { REQUEST generator delay, GRANT processor delay, } \\
\text { Scheduling delay }\end{array}$ \\
\hline BS & $\begin{array}{l}\text { REQUEST processor delay, REPORT generator delay, } \\
\text { GATE processor delay (wavelength), GATE processor delay } \\
\text { (bandwidth), GRANT generator delay, QoS mapping delay, } \\
\text { Queuing delay, Scheduling delay }\end{array}$ \\
\hline ONU & $\begin{array}{l}\text { REPORT processor delay, REPORT generator delay, GATE } \\
\text { processor delay (wavelength), GATE processor delay } \\
\text { (bandwidth) Wavelength allocation algorithm delay, }\end{array}$ \\
& $\begin{array}{l}\text { Bandwidth allocation algorithm delay, GATE generator } \\
\text { delay (wavelength), GATE generator delay (bandwidth), } \\
\text { Queuing delay }\end{array}$ \\
\hline OLT & $\begin{array}{l}\text { REPORT processor delay, Wavelength allocation algorithm } \\
\text { delay, Bandwidth allocation algorithm delay, GATE } \\
\text { generator delay (wavelength), GATE generator delay } \\
\text { (bandwidth) }\end{array}$ \\
\hline
\end{tabular}




\begin{tabular}{|c|c|c|}
\hline Module & Module name & Converged Scenario (Full Dynamic) (Each module runs once per service cycle) \\
\hline (2.a) & $\begin{array}{l}\text { OLT }-> \\
\text { allocation }\end{array}$ & $\begin{array}{l}\text { This module runs once in every service cycle in order to allocate a wavelength id from the OLT to a given ONU. The } \\
\text { allocated wavelength may change per service cycle based on the load per channel and the total network load taking } \\
\text { into account the available wavelengths on the OLT and the supported wavelengths on the OLT and a given ONU. }\end{array}$ \\
\hline (2.b) & OLT -> REPORT processor & $\begin{array}{l}\text { This module runs once in every service cycle in order to capture the queue status of a given ONU which may be } \\
\text { different in every service cycle based on the dynamic nature of the Internet traffic. }\end{array}$ \\
\hline (2.c) & $\begin{array}{l}\text { OLT } \quad>\quad \text { bandwidth } \\
\text { allocation }\end{array}$ & $\begin{array}{l}\text { This module runs once in every service cycle in order to allocate bandwidth from the OLT to a given ONU by taking } \\
\text { in to account the actual need of a given ONU (captured from module } 2 . b \text { ), traffic status on other ONUs (network } \\
\text { traffic) and bandwidth allocation boundaries e.g. the minimum guaranteed bandwidth for each ONU and maximum } \\
\text { transmission window. }\end{array}$ \\
\hline$(2 . d)$ & $\begin{array}{l}\text { OLT -> GATE generator } \\
\text { (wavelength) }\end{array}$ & $\begin{array}{l}\text { This module runs once per service cycle in order to insert the wavelength allocation decisions (module 2.a outputs) } \\
\text { inside the MPCP Ext. GATE messages and immediately goes to module 2.e. }\end{array}$ \\
\hline$(2 . e)$ & $\begin{array}{l}\text { OLT -> GATE generator } \\
\text { (bandwidth) }\end{array}$ & $\begin{array}{l}\text { This module runs once per service cycle in order to insert the bandwidth allocation decisions (module 2.c outputs) } \\
\text { inside the MPCP Ext. GATE messages which will broadcast immediately to all the ONUs. }\end{array}$ \\
\hline (8) & BS-> REPORT generator & $\begin{array}{l}\text { This module runs once per service cycle in order to report the queue status of a given BS (buffer length) to the } \\
\text { associated ONU. The reported queue status may be changed per service cycle based on the dynamic nature of the } \\
\text { Internet traffic. }\end{array}$ \\
\hline (9.a) & ONU-> REPORT generator & $\begin{array}{l}\text { This module runs once per service cycle in order to update the OLT about the buffer length (queue status) which is } \\
\text { associated with a given ONU. }\end{array}$ \\
\hline (9.b) & $\begin{array}{l}\text { ONU-> GATE processor } \\
\text { (wavelength) }\end{array}$ & $\begin{array}{l}\text { This module runs once per service cycle in order to disclose the channel ID allocated from the OLT to a given ONU } \\
\text { which may be different in each service cycle based on the network load, channel supports and availability as well as } \\
\text { the waveband boundaries. }\end{array}$ \\
\hline (9.c) & $\begin{array}{l}\text { ONU-> GATE processor } \\
\text { (bandwidth) }\end{array}$ & $\begin{array}{l}\text { This module runs once per service cycle in order to reveal the amount of the allocated bandwidth from the OLT on } \\
\text { associated channel ID (outputs from module 9.b) along with the transmission start-time and transmission duration for } \\
\text { a given ONU. }\end{array}$ \\
\hline (10.a) & $\begin{array}{l}\text { ONU-> } \\
\text { allocation }\end{array}$ & $\begin{array}{l}\text { This module runs once per service cycle in order to allocate a channel ID from the ONU to a given BS which may be } \\
\text { different in each service cycle based on the load on a given BS, total channel load, supported channels and available } \\
\text { channels. }\end{array}$ \\
\hline (10.b) & ONU-> REPORT processor & $\begin{array}{l}\text { This module runs once per service cycle in order to unveil the buffer length (queue status) associated with a given BS } \\
\text { in a given service cycle before deciding any bandwidth allocations from a given ONU to the associated BSs. }\end{array}$ \\
\hline$(10 . c)$ & $\begin{array}{l}\text { ONU-> } \\
\text { allocation }\end{array}$ & $\begin{array}{l}\text { This module runs once per service cycle in order to allocate bandwidth (channel portion) from a given ONU to the } \\
\text { associated BSs based on the bandwidth allocation boundaries, available bandwidth as well as the queue status of a } \\
\text { given BS (output from module 10.b). }\end{array}$ \\
\hline (11.a) & $\begin{array}{l}\text { ONU-> GATE generator } \\
\text { (wavelength) }\end{array}$ & $\begin{array}{l}\text { This module runs once per service cycle in order to insert the allocated channel ID (outputs from module 10.a) into } \\
\text { the MPCP Ext. GATE messages and then goes straight to module 11.b. }\end{array}$ \\
\hline (11.b) & $\begin{array}{l}\text { ONU-> GATE generator } \\
\text { (bandwidth) }\end{array}$ & $\begin{array}{l}\text { This module runs once per service cycle in order to insert the bandwidth allocation decisions from the ONU (outputs } \\
\text { from module 10.c) inside the MPCP Ext. GATE messages, which also carry the allocated channel id for a given BS } \\
\text { (inserted by module 11.a). The generated GATE messages will then broadcast immediately to BSs of a given ONU. }\end{array}$ \\
\hline (12.a) & $\begin{array}{l}\text { BS-> GATE processor } \\
\text { (wavelength) }\end{array}$ & $\begin{array}{l}\text { This module runs once per service cycle in order to disclose the allocated channel id assigned from a given ONU to } \\
\text { the associated BS by module 10.a during the latest service cycle. }\end{array}$ \\
\hline (12.b) & $\begin{array}{l}\text { BS-> GATE processor } \\
\text { (bandwidth) }\end{array}$ & $\begin{array}{l}\text { This module runs once per service cycle in order to capture the allocated amount of bandwidth, transmission start- } \\
\text { time, transmission duration as well as the occupied MAC address assigned from a given ONU (outputs from module } \\
\text { 10.c) to the associated BS. }\end{array}$ \\
\hline
\end{tabular}

\section{ACKNOWLEDGMENT}

The author would wish to acknowledge the support of the University of Ulster and University of Abertay Dundee for funding this work.

\section{REFERENCES}

[1] ITU-T G.9S4.x (GPON), at: http://www.itu.int/rec/T-REC-G/e [retrieved: June, 2013]

[2] ITU-T G.983.1 (BPON), at: http://www.itu.int/rec/T-REC-G.983.1200501-I/en [retrieved: June, 2013]

[3] IEEE 802.3ah (EPON)at: http://www.ieee802.org/3/ah/index.html [retrieved: June, 2013]

[4] G. Kramer, B. Mukherjee, and G. Perawnto, "Ethernet PON (ePON): Design and Analysis of an Optical Access Network," Photonic Network Communications, vol. 3, no. 3, July. 2001, pp. 307-19.

[5] D. J. Shin, D. K. Jung, H. S. Shin, J. W. Kwon, S. Hwang, Y. Oh, and C. Shim, "Hybrid WDM/TDM-PON with Wavelength-SelectionFree Transmitters," Journal of Lightwave Technology, vol. 23, no. 1, January. 2005, pp. 187-194.

[6] A. Banerjee, Y. Park, F. Clarke, H. Song, S. Yang, G. Kramer, K. Kim, and B. Mukherjee, "Wavelength-division multiplexed passive optical network (WDM-PON) technologies for broadband access-A review [Invited]," OSA Journal of Optical Networking - Special Issue Optical Access Networks, vol. 4, no. 11, November. 2005, pp. 737758.

[7] C. Eklund, R. B. Marks, K. L. Stanwood, and S. Wang, "IEEE Standard 802.16: A Technical Overview of the Wireless MAN Air
Interface for Broadband Wireless Access," IEEE Communications Magazine, vol. 40, no.6, 2002, pp. 98-107.

[8] IEEE Standard for Local and Metropolitan Area Networks Part 16 : Air Interface for Fixed Broadband Wireless Access Systems, 2004

9] G. Shen et al, "Fixed Mobile Convergence Architectures for Broadband Access: Integration of EPON and WiMAX," IEEE Commun. Mag., Volume 45, Issue 8, August 2007, pp. 44 - 50.

[10] S. Sarkar et al., "Hybrid wireless-optical broadband- access network (WOBAN): A review of relevant challenges," JLT, Nov. 2007, Vol. 25, No. 11, pp. 3329-3340.

[11] W.-T. Shaw et al., "Hybrid Architecture and Integrated Routing in a Scalable Optical-Wireless Access Network,” JLT. vol. 25, no. 11, Nov. 2007, pp. 3443-3451.

[12] A. Ahmed et al., "A New Bandwidth Allocation Algorithm for EPON-WiMAX Hybrid Access Networks," IEEE Globecom, 2010.

[13] Y. Kun et al., "Convergence of Ethernet PON and IEEE 802.16 broadband access networks and its QoS-aware dynamic bandwidth allocation scheme," IEEE J. Sel. Areas Commun., vol. 27, no. 2, 2009, pp. 101-116.

[14] S. Ou et al., "Integrated Dynamic Bandwidth Allocation in Converged Passive Optical Networks and IEEE 802.16 Networks," IEEE Sys. J., Vol. 4, No. 4, Dec. 2010, pp. 467- 476.

[15] B. Jung et al., "Centralized Scheduling Mechanism for Enhanced End-to-End Delay and QoS Support in Integrated Architecture of EPON and WiMAX", JLT, vol. 28, no. 16, Aug.2010.

[16] Y.Luo et al., "Integrating Optical and Wireless Services in the Access Network," OSA, 2006.

[17] M. McGarry et al., "WDM Ethernet passive optical networks, "IEEE Optical Communications, Vo1.44, Issue2, Feb.2006, S18-S25. 